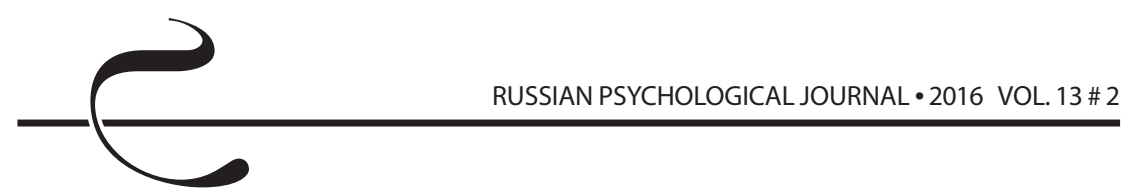

для дискУссии

УДК 159.9.015

\title{
СЛОЖНЫЕ СИСТЕМЫ В ПСИХОФИЗИОЛОГИИ ПРЕДСТАВЛЯЮТ ЭФФЕКТ «ПОВТОРЕНИЕ БЕЗ ПОВТОРЕНИЙ» Н. А. БЕРНШТЕЙНА
}

\author{
Еськов Валерий Матвеевич \\ Зинченко Юрий Петрович \\ Веракса Александр Николаевич \\ Филатова Диана Юрьевна
}

Продолжается дискуссия об особых свойствахживых систем - complexity (2омеостатических систем). Представлено новое понимание сотрlexity и систем mpemьего muna W. Weaver в рамках новой теории хаоса-самоорганизации и постнеклассики В. С. Стёпина. Сейчас мы демонстрируем отсутствие эффективности в применении стохастики и детерминированного хаоса (которые были представлены I. R. Prigogine, J. A. Wheeler u M. Gell-Mann за последние 50 лет) для описания сложных биосистем.

Возникает возможность нового представления о гомеостатических системах. Эти системы находятся в непрерывном хаотическом движении (неопределенность 2-го типа), и для них методы статистики применять нецелесообразно. Обозначается граница современной детерминистской и стохастической науки в описании сложных гомеостатических биосистем. Это знаменует и новый этап развития философии науки и науки о живой nрироде (complexity) в целом. В основе этого разделения - эффект «повторение без повторений» Н. А. Бернштейна, который сейчас количественно описывается эфрфектом Еськова - Зинченко.

Ключевые слова: хаос, стохастика, самоорганизачия, гомеостаз, биомеханика, эффект Еськова - Зинченко, гомеостатические системы, теория хаоса-самоорганизации, СТT (системы третьего типа).

\section{Введение}

Развитие человечества невозможно без понимания и изучения особенностей живых систем (системы третьего muna - CTT - complexity) как уникальных систем, выходящих за рамки традиционной детерминистской, стохастической науки и теории детерминированного хаоса. Впервые об этом 
было представлено в работах Н. А. Бернштейна [2] («повторение без повторений») в биомеханике (1947) и в публикации W. Weaver о системах третьего типа [22]. H. Haken при создании синергетики очень близко подошел к описанию специфики живых систем, но он не вышел за рамки традиционной науки [20]. Сейчас, с созданием третьей парадигмы, это означает отсутствие реальных моделей особых живых систем - СТТ. В рамках современной науки любая модель СТТ имеет историческое значение, следующая динамика (выборка) произвольно не повторима, и тогда традиционная детерминистская или стохастическая модель описывает только артефакты (разовые, уникальные процессы). Повторить динамику СТТ невозможно, любые статистические характеристики СТТ - complexity будут непрерывно изменяться [3, 7, 8, 9, 15, 16, 17]. Все это представляет количественную основу эффекта Еськова - Зинченко, который показал хаотическую природу биомеханических систем.

В разрабатываемой сейчас теории хаоса-самоорганизации (TXC) наши научные подходы отрицают возможность применения при описании СТТ любой функциональной зависимости $y=y(x)$ и использования статистических методов расчета параметров живых систем. Любая модель для описания СТТ в виде $y=f(x)$, статистических функций распределения $f(x)$, спектральных плотностей сигнала (СПС), автокорреляционных функций $A(t)$, фрактальной размерности и других характеристик процесса имеет разовое (историческое) значение для сложных биомеханических систем. СТТ - complexity, к которым в первую очередь принадлежат функциональные системы организма (ФСО) по П. К. Анохину [2], не могут демонстрировать стационарных режимов своего вектора состояния (BCC) $x=x(t)=\left(x_{1}, x_{2}, \ldots, x_{m}\right)^{T}$ в фазовом пространстве состояний (ФПС). Все это означает, что невозможно повторить начальные состояния системы $x\left(t_{0}\right)$ произвольно, как и любые другие состояния $x(t)$ в ФПС $[4,5,10,13,14]$. Количественная характеристика такой неповторимости и составляет основу эффекта Еськова - Зинченко.

С позиций ТХС очевидно, что модели и методы СТТ нарушают главный принцип современной науки - повторяемость динамики (или состояний) биомеханических систем. В рамках традиционной науки мы должны иметь повторяющиеся процессы! Если нет произвольного повторения начального состояния СТТ в виде $x\left(t_{0}\right)$ и любого другого состояния системы, если мы не можем произвольно «повторить» вектор состояния системы $x=x(t)=\left(x_{1}, x_{2}, \ldots, x_{m}\right)^{\top}$ в данной точке фазового пространства состояний (ФПС), то мы имеем дело с уникальными системами. По определению I. R. Prigogine, они не являются объектом современной науки [21], и их невозможно описывать функциями $y(x)$ или функциями распределения $f(x)$ в ФПС $[15,16,17,18]$. Тогда возникает необходимость создания новых подходов в науке в целом. 


\section{Особенности СТT - complexity}

Высказывание об уникальности биосистем впервые представил I. R. Prigogine в своем предсмертном обращении к потомкам ("The Die is not (ast"), уже тогда нобелевский лауреат был твердо уверен, что СТТ complexity (живые системы) не являются объектом современной (традиционной) науки [21]. Но другую науку И. Р. Пригожин не создал. Его термодинамика неравновесных систем (THC) тоже не имеет прямого отношения к живым системам (СТT - complexity). Было показано, что теорема Пригожина Гленсдорфа о минимуме скорости $P=d E / d t$ изменения энтропии $E$ для СТТ не выполняется $[15,16,17,18]$. Термодинамика И. Р. Пригожина не описывает сложные биосистемы - СТТ - вблизи равновесия, т. к. энтропия вообще может не изменяться существенно при переходе СТТ от равновесного состояния к неравновесному [5, 8, 10, 13, 18].

Роджер Пенроуз отмечал: “Что означает "вычислимость", когда в качестве входных и выходных данных допускаются непрерывно изменяющиеся nараметры?» [см. 11, с. 165]. Для уникальных систем невозможно произвольно повторить начальное состояние $x\left(t_{0}\right)$ и любое другое $x(t)$ тоже. Для СТТ невозможно повторить конечное состояние $x\left(t_{k}\right)$ ни в виде статистических функций $f(x)$, их $C П C, A(t)$, фракталов и т. д. Для любой гомеостатической системы - СТТ (complexity) нужна другая наука - это наука о гомеостатических системах с максимальной неопределенностью их динамики. Такую науку мы сейчас создаем - это теория хаоса-самоорганизации - ТХС. Она основана на расчетах квазиаттракторов - KА $[1,4,5,8,9,10]$ и на новом использовании нейроэмуляторов (НЭВМ) $[13,14]$. Тогда повторяются параметры квазиаттракторов и определяются параметры порядка $x_{i}$ для всего BCC $x(t)[15,16,17,18]$.

Работы Н. А. Бернштейна [6], W. Weaver [22] и I. R. Prigogine [21] (в их попытках изучать и описывать (ТT - complexity) были лишь началом и призывом к изучению таких особых биосистем. СТТ - complexity изучать в рамках традиционной науки как уникальные СТТ весьма затруднительно (если вообще возможно). Гомеостатические системы имеют две неопределенности 1-го и 2-го типов (здесь статистика не работает!). Для их описания можно использовать компартментно-кластерную теорию биосистем (ККТБ), нейроэмуляторы и ТХC $[15,16,17,18]$. В последнем случае мы имеем определенность параметров квазиаттракторов для неопределенных, гомеостатических систем третьего типа, с которыми приходится работать в психофизиологии. Квазиаттракторы могут описывать динамику СТТ - complexity, но понятие стационарных режимов (гомеостаза) и кинематики СТТ в ФПС имеет другой смысл и другие модели $[13,14,15,16,17,18]$, которые представляют эффект Еськова - Зинченко в психофизиологии. 
Первая статья И. Р. Пригожина (Философия нестабильности, 1991) из целой серии публикаций о неопределенности и неустойчивости систем была лишь прелюдией к познанию жизни, но за эту четверть века, фактически, никакой реакции на эту публикацию не последовало (за исключением комментария С. П. Курдюмова). Наука на современном этапе ее развития становится инертной, она слабо откликается на новые факты. Возникает иллюзия, что человечество все уже изучило и познало, но это далеко не так! Мы живем в мире иллюзий о гомеостатичности психофизиологических и любых биосистем в целом, в психологии и психофизиологии в целом, но их динамика особая. Нужны и другие методы изучения СТT - complexity $[1,3,4,5,7,8,9,10]$.

Сейчас наблюдается очень слабая реакция на необычные свойства CTT - complexity, которые Н. А. Бернштейн пытался представить почти 70 лет назад $[2,8,9]$. Наука в целом достигла некоторого уровня и как бы не желает дальше развиваться, т. е. развиваются пока прикладные аспекты, и очень мало средств и внимания уделяется перспективам развития науки, общего познания мира. Создание современной научной картины мира не может базироваться на мире научных иллюзий и на искаженных представлениях о живых системах как о системах детерминистских или стохастических. Даже детерминированный хаос для СТТ - это тоже иллюзия. Мир живых систем, СТT - complexity $[15,16,17,18]$ - это другой мир, мир нестабильности и хаотических систем, о котором пытался сказать И. Р. Пригожин (но у него отсутствовал математический аппарат для описания СТТ). Н. А. Бернштейн представлял как минимум четыре регуляторные системы в организации движений (A, B, C, D) и тоже пытался об этом сказать. Однако в эффекте Еськова - Зинченко мы дали этому количественную оценку.

Сейчас наука глубоко уверовала, что биосистемы являются объектом детерминированного или стохастического подходов (в крайнем случае детерминированного хаоса). Однако реальность совершенно другая - живые системы не являются объектом современной науки. Об этом пытались сказать Н. А. Бернштейн, W. Weaver, I. R. Prigogine, но на их высказывания никто не обращал внимания (включая и представителей философии науки). Однако уже сейчас мы подходим к переосмысливанию новых научных фактов и новых методов оценки динамики (и статики - гомеостатики) сложных биосистем - complexity. Возникли границы наших возможностей в рамках современной науки научного познания мира живых систем (и не только!). В первую очередь это касается психологии и психофизиологии, где управление движением происходит при участии миллиардов нейронов, каждый из которых сам уже не может стохастически работать.

Современная наука, требующая повторений любых процессов и явлений, совершенно бессильна перед неопределенностью и неповторяемостью 
живых систем - СТТ. Более того, оказалось, что гомеостатическими свойствами обладают не только живые системы. Это и системы регуляции климата, метеорологические параметры окружающей среды пребывания человека. Самоорганизацией обладает и вся Вселенная. Климат, погода, организм человека и социумы (человечество в целом) являются гомеостатическими системами. Их динамика на любом временном отрезке времени $\Delta t$ не может быть произвольно повторена [3, 6, 7, 8, 9, 10].

Динамика гомеостатических систем имеет особые параметры, которые не укладываются в обычные статистические критерии ( $f(x), C П C, A(t)$ непрерывно и хаотически изменяются). К гомеостатическим системам невозможно применять и термодинамический подход, т. к. энтропия СТТ отлична от энтропии молекулярных систем. У живых систем другие критерии статичности (неизменности) и другие критерии кинематики. Мы сейчас в рамках ТХС рассматриваем эволюцию СТТ - complexity как движение квазиаттракторов в ФПС, и это движение отлично от обычного механического движения, которое изучается в физике. В целом, для СТТ - complexity мы разработали новые критерии стационарности (гомеостатичности), новые критерии кинематики и новые термодинамические подходы [3, 4, 5, 7, 8, 9, 10, 13, 14, 15, 16, 17, 18].

СТТ демонстрируют полную инверсию понятий. Что в физике является движением, когда $d x / d t \neq 0$ и наблюдается изменение фазовых координат $x_{i}$, то в TXC, наоборот, может представлять статику (гомеостатичность). В этом случае нет существенных изменений параметров квазиаттракторов, не изменяются их объемы $V_{G}$ и координаты их центров $x_{i}^{c}$. Покой в ТХС соответствует непрерывному движению $x(t)$ в ФПС. Мы имеем инверсию понятий движения, и это требует научного переосмысливания и новых моделей и методов расчета. Более того, в ТХС вводится новое понятие скорости движения квазиаттракторов, которое описывает эволюцию сложных биосистем (СТТ - complexity).

Более того, сейчас создается новая, третья парадигма естествознания и ее теоретическая основа - ТХC [3]. Третья парадигма естествознания и теория хаоса-самоорганизации изучают и представляют живые системы и все СТТ в целом [3, 12]. Можно выделить несколько ключевых понятий и условий, методов для представления СТТ. Во-первых, большой набор фактов и доказательств того, что СТТ не являются объектами современной детерминистско-стохастической науки из-за непрерывной и хаотической изменчивости параметров СТТ $x_{i}$. Во-вторых, разработаны новые методы и модели, которые способны описывать статику и кинематику, т. е. гомеостаз и эволюцию, таких особых CTT - complexity. B-mpemьux, в различных периодических изданиях (более 400 статей и докладов) и монографиях (более 40 книг) показано практическое применение в биологии, экологии, медицине разработанных 
методов для оценки состояния организма человека, климата, погоды и т. д. Рассмотрим теперь особенности хаоса в организации движений более подробно, на примере кластера «доказательство» в изучении эффекта Н. А. Бернштейна «повторение без повторений», который сейчас получил количественное подтверждение в виде эффекта Еськова - Зинченко в психофизиологии и биомеханике.

\section{Особые свойства СТТ в биомеханике как основа эффекта Еськова - Зинченко}

В третьей парадигме и теории хаоса-самоорганизации вводится пять основных принципов (свойств) организации особых биосистем (СТТ complexity) $[3,8]$. Еще раз отметим, что все особые свойства биосистем были получены с помощью многочисленных наблюдений и экспериментов с более чем 20000 испытуемых (и более 1 млн. выборок). При этом регистрировались не только треморограммы (ТМГ) и теппинграммы (ТПГ) в биомеханике и физиологии нервно-мышечной системы (HМC), но и при анализе электромиограмм (ЭМГ), электроэнчефалограмм (ЭЭГ), электронейрограмм (ЭНГ) в электрофизиологии; кардиоинтервалов (КИ) и электрокардиограмм (ЭКГ) при изучении реакций кардиореспираторной системы (КРС) как важнейшей функциональной системы организма (ФСО) человека $[13,14,15,16,17,18]$. Наблюдалась динамика ряда биохимических показателей (например, при инфекционном гепатите A, B, C и изменениях уровня сахара в крови при метаболических нарушениях) - БХП.

Все такие переменные можно кратко представить как некоторую одну переменную $x_{1}(t)$ (для каждого случая). Для таких $x_{1}(t)$ можно получить скорость их изменения, т. е. $x_{2}(t)=d x_{1}(t)$, и ускорение $x_{3}=d x_{2} / d t[5,15]$. В этом случае мы переходим к трехмерному подпространству $x_{i}(t)=\left(x_{i 1}, x_{i 2}, x_{i 3}\right)^{T}$, где $x_{i}$ - любая координата всего m-мерного пространства состояний вектора $x(t)$, а подпространство содержит саму координату $x_{i 1}$, ее скорость $x_{i 2}$ и ускорение $x_{i 3}$.

Для любой переменной (ТМГ, ТПГ, ЭМГ, ЭЭГ, КИ, БХП) мы можем построить фазовое пространство состояний (ФПС) вектора $x_{i}^{2}=\left(x_{i 1}, x_{i 2}\right)^{\top}$ или $x_{i}^{3}=\left(x_{i 1}, x_{i 2}, x_{i 3}\right)^{T}$, и в нем (ФПС) изучать характер движения $x_{i}(t)$. Если система находится в гомеостазе, то квазиаттрактор (объем $V_{G}$, внутри которого непрерывно и хаотически движется $x(t))$ будет сохранять свои параметры в виде самого объема $V_{G}$ и координат его центра $x_{i}^{c}$ для каждой $i$-й координаты и всего вектора $x(t)[15,16]$. Это является главной характеристикой гомеостаза. С позиций детерминизма мы имеем непрерывное движение вектора состояния $x(t)$ (хаос траектории, $d x / d t \neq 0$ постоянно), а с позиций ТХС мы имеем покой (отсутствие изменений параметров квазиаттракторов). 
Мы построили несколько сотен матриц парного сравнения выборок $x_{i}$ для всех перечисленных выше переменных и везде имели малое число $k$ совпадений выборок. Обычно $k<20$ \% или даже 5 \% от общего числа пар сравнения $[8,9,13,14]$.

С позиций ТХC очень важно подчеркнуть, что из-за непрерывного и хаотического изменения $x(t)$ в ФПС мы не можем использовать статистику в оценке СТТ. Любое измерение в психологии и психофизиологии не дает объективного представления о состоянии системы, т. к. на другом интервале времени $\Delta t$ мы получим другие выборки $x_{i}$ и другие функции распределения $f\left(x_{i}\right)$. Все непрерывно изменяется для одной и той же системы, находящейся в неизменном гомеостазе. Для гомеостатических систем характерен калейдоскоп не только функций $f(x)$, но и спектральных плотностей сигнала (СПС) всех $x_{i}$ их автокорреляционных функций $A(t)$ и других характеристик и параметров $x(t)$, которые рассчитываются в рамках стохастики (включая и фрактальные размерности). В описании и изучении медико-биологических систем методы и модели традиционной науки дают низкую эффективность. С нашей точки зрения, биология, медицина и психофизиология до настоящего времени использовали разовые измерения, разовые выборки, которые не могли объективно представлять состояния биосистем (СТT) $[5,7,15]$. Наступает время третьей парадигмы, ТХС и объективной оценки гомеостаза и эволюции сложных биосистем - complexity, учета их непрерывного и хаотического движения вектора $x(t)$ в ФПС, когда непрерывно все изменяется $[3,4,5,7,8,9,10,15]$. При этом возникает проблема объективного измерения неизменности психического состояния испытуемых (что реально изменяется?).

Для иллюстрации сказанному представим три таблицы - матрицы парного сравнения выборок испытуемого ГДВ, находящегося в одинаковом психическом состоянии (но статистика не дает такой одинаковости). В таблице 1 мы демонстрируем матрицу парных сравнений 15-ти треморограмм (ТМГ), полученных от одного испытуемого (подряд), здесь $k_{1}=3$. В таблице 2 мы показываем матрицу парных сравнений 15-ти выборок теппинграмм (ТПГ), здесь $k_{2}=17$. Наконец, в таблице 3 мы показываем матрицу парных сравнений 15-ти автокорреляционных функций $A(t)$ этого же испытуемого $k_{3}=27$.

Очевидно, что $k_{1}$ (тремор) существенно отличается от $k_{2}$ (ТПГ). Закономерно, что все ТПГ всегда показывали четырех- или пятикратное превышение над ТМГ. Этим непроизвольное движение (тремор) отличается от произвольного движения (теппинг). Но соотношение $k_{1}$ и $k_{2}$ повторяются для всех испытуемых. Характерно, что $A(t)$ не стремится к нулю с ростом времени $t$, и всегда могут быть совпадения даже между 1-й и 15-й выборками, т. е. автокорреляция $A(t)$ не стремится к нулю при $t \rightarrow \infty$. 
Таблица 1.

Матрица парного сравнения выборок треморограмм испытуемого ГДВ (число повторов $N=15$ ), использовался критерий Вилкоксона (уровень значимости $p<0.05$, число совпадений $k_{1}=3$ )

\begin{tabular}{|c|c|c|c|c|c|c|c|c|c|c|c|c|c|c|c|}
\hline & 1 & 2 & 3 & 4 & 5 & 6 & 7 & 8 & 9 & 10 & 11 & 12 & 13 & 14 & 15 \\
\hline 1. & & 0.00 & 0.00 & 0.02 & 0.00 & 0.00 & 0.00 & 0.00 & 0.00 & 0.00 & 0.00 & 0.00 & 0.96 & 0.00 & 0.00 \\
\hline 2. & 0.00 & & 0.00 & 0.00 & 0.00 & 0.00 & 0.00 & 0.00 & 0.00 & 0.00 & 0.00 & 0.00 & 0.00 & 0.00 & 0.00 \\
\hline 3. & 0.00 & 0.00 & & 0.00 & 0.00 & 0.00 & 0.00 & 0.00 & 0.00 & 0.00 & 0.00 & 0.00 & 0.00 & 0.00 & 0.00 \\
\hline 4. & 0.02 & 0.00 & 0.00 & & 0.00 & 0.00 & 0.00 & 0.00 & 0.00 & 0.00 & 0.00 & 0.00 & 0.00 & 0.00 & 0.00 \\
\hline 5. & 0.00 & 0.00 & 0.00 & 0.00 & & 0.00 & 0.00 & 0.00 & 0.00 & 0.00 & 0.00 & 0.00 & 0.00 & 0.00 & 0.00 \\
\hline 6. & 0.00 & 0.00 & 0.00 & 0.00 & 0.00 & & 0.00 & 0.00 & 0.00 & 0.00 & 0.00 & 0.00 & 0.00 & 0.00 & 0.00 \\
\hline 7. & 0.00 & 0.00 & 0.00 & 0.00 & 0.00 & 0.00 & & 0.00 & 0.00 & 0.00 & 0.00 & 0.00 & 0.00 & 0.15 & 0.00 \\
\hline 8. & 0.00 & 0.00 & 0.00 & 0.00 & 0.00 & 0.00 & 0.00 & & 0.42 & 0.00 & 0.00 & 0.00 & 0.00 & 0.00 & 0.00 \\
\hline 9. & 0.00 & 0.00 & 0.00 & 0.00 & 0.00 & 0.00 & 0.00 & 0.42 & & 0.00 & 0.00 & 0.00 & 0.00 & 0.00 & 0.00 \\
\hline 10. & 0.00 & 0.00 & 0.00 & 0.00 & 0.00 & 0.00 & 0.00 & 0.00 & 0.00 & & 0.00 & 0.00 & 0.00 & 0.00 & 0.00 \\
\hline 11. & 0.00 & 0.00 & 0.00 & 0.00 & 0.00 & 0.00 & 0.00 & 0.00 & 0.00 & 0.00 & & 0.00 & 0.00 & 0.00 & 0.00 \\
\hline 12. & 0.00 & 0.00 & 0.00 & 0.00 & 0.00 & 0.00 & 0.00 & 0.00 & 0.00 & 0.00 & 0.00 & & 0.00 & 0.00 & 0.00 \\
\hline 13. & 0.96 & 0.00 & 0.00 & 0.00 & 0.00 & 0.00 & 0.00 & 0.00 & 0.00 & 0.00 & 0.00 & 0.00 & & 0.00 & 0.00 \\
\hline 14. & 0.00 & 0.00 & 0.00 & 0.00 & 0.00 & 0.00 & 0.15 & 0.00 & 0.00 & 0.00 & 0.00 & 0.00 & 0.00 & & 0.00 \\
\hline 15. & 0.00 & 0.00 & 0.00 & 0.00 & 0.00 & 0.00 & 0.00 & 0.00 & 0.00 & 0.00 & 0.00 & 0.00 & 0.00 & 0.00 & \\
\hline
\end{tabular}


Таблица 2.

Матрица парного сравнения выборок теппинграмм испытуемого ГДВ (число повторов $N=15$ ), использовался критерий Вилкоксона (уровень значимости $p<0.05$, число совпадений $k_{2}=17$ )

\begin{tabular}{|c|c|c|c|c|c|c|c|c|c|c|c|c|c|c|c|}
\hline & $\mathbf{1}$ & $\mathbf{2}$ & $\mathbf{3}$ & $\mathbf{4}$ & $\mathbf{5}$ & $\mathbf{6}$ & $\mathbf{7}$ & $\mathbf{8}$ & $\mathbf{9}$ & $\mathbf{1 0}$ & $\mathbf{1 1}$ & $\mathbf{1 2}$ & $\mathbf{1 3}$ & $\mathbf{1 4}$ & $\mathbf{1 5}$ \\
\hline 1. & & 0.00 & 0.00 & 0.00 & 0.00 & $\mathbf{0 . 1 7}$ & 0.00 & 0.00 & 0.00 & 0.00 & 0.00 & 0.00 & 0.00 & 0.01 & 0.00 \\
\hline 2. & 0.00 & & 0.00 & 0.00 & $\mathbf{0 . 2 6}$ & 0.00 & 0.00 & 0.00 & 0.00 & 0.00 & 0.00 & $\mathbf{0 . 9 4}$ & 0.04 & 0.00 & 0.00 \\
\hline 3. & 0.00 & 0.00 & & 0.00 & 0.00 & 0.00 & $\mathbf{0 . 7 4}$ & 0.00 & 0.00 & 0.00 & $\mathbf{0 . 4 5}$ & $\mathbf{0 . 0 5}$ & 0.00 & 0.00 & 0.00 \\
\hline 4. & 0.02 & 0.00 & 0.00 & & 0.00 & 0.00 & 0.00 & $\mathbf{0 . 5 2}$ & $\mathbf{0 . 1 4}$ & $\mathbf{0 . 1 8}$ & 0.00 & 0.00 & 0.00 & 0.00 & 0.03 \\
\hline 5. & 0.00 & 0.26 & 0.00 & 0.00 & & 0.00 & 0.00 & 0.00 & 0.00 & 0.00 & 0.00 & 0.03 & $\mathbf{0 . 3 8}$ & 0.02 & 0.00 \\
\hline 6. & 0.17 & 0.00 & 0.00 & 0.00 & 0.00 & & 0.00 & 0.00 & 0.00 & 0.00 & 0.00 & 0.00 & 0.00 & $\mathbf{0 . 1 0}$ & 0.00 \\
\hline 7. & 0.00 & 0.00 & 0.74 & 0.00 & 0.00 & 0.00 & & 0.00 & 0.00 & 0.00 & $\mathbf{0 . 7 4}$ & 0.04 & 0.00 & 0.00 & 0.00 \\
\hline 8. & 0.00 & 0.00 & 0.00 & 0.52 & 0.00 & 0.00 & 0.00 & & $\mathbf{0 . 0 6}$ & $\mathbf{0 . 9 6}$ & 0.00 & 0.00 & 0.00 & 0.00 & 0.00 \\
\hline 9. & 0.00 & 0.00 & 0.00 & 0.14 & 0.00 & 0.00 & 0.00 & 0.06 & & $\mathbf{0 . 8 2}$ & 0.00 & 0.00 & 0.00 & 0.00 & 0.01 \\
\hline 10. & 0.00 & 0.00 & 0.00 & 0.18 & 0.00 & 0.00 & 0.00 & 0.96 & 0.82 & & 0.00 & 0.00 & 0.00 & 0.00 & $\mathbf{0 . 1 5}$ \\
\hline 11. & 0.00 & 0.00 & 0.45 & 0.00 & 0.00 & 0.00 & 0.74 & 0.00 & 0.00 & 0.00 & & 0.04 & 0.00 & 0.00 & 0.00 \\
\hline 12. & 0.00 & 0.94 & 0.05 & 0.00 & 0.03 & 0.00 & 0.04 & 0.00 & 0.00 & 0.00 & 0.04 & & 0.02 & 0.00 & 0.00 \\
\hline 13. & 0.00 & 0.04 & 0.00 & 0.00 & 0.38 & 0.00 & 0.00 & 0.00 & 0.00 & 0.00 & 0.00 & 0.02 & & $\mathbf{0 . 0 6}$ & 0.00 \\
\hline & 0.01 & 0.00 & 0.00 & 0.00 & 0.02 & 0.10 & 0.00 & 0.00 & 0.00 & 0.00 & 0.00 & 0.00 & 0.06 & & 0.00 \\
\hline
\end{tabular}


Таблица 3.

Матрица парного сравнения 15-ти автокорреляционных функций $A(t)$ треморограмм одного испытуемого ГДВ при повторных экспериментах $\left(k_{3}=27\right)$, по критерию Вилкоксона (для непараметрического распределения)

\begin{tabular}{|c|c|c|c|c|c|c|c|c|c|c|c|c|c|c|c|}
\hline & 1 & 2 & 3 & 4 & 5 & 6 & 7 & 8 & 9 & 10 & 11 & 12 & 13 & 14 & 15 \\
\hline 1. & & 0.00 & 0.00 & 0.00 & 0.00 & 0.00 & 0.00 & 0.00 & 0.00 & 0.00 & 0.00 & 0.00 & 0.00 & 0.00 & 0.00 \\
\hline 2. & 0.00 & & 0.01 & 0.00 & 0.00 & 0.07 & 0.01 & 0.00 & 0.00 & 0.00 & 0.93 & 0.00 & 0.21 & 0.00 & 0.02 \\
\hline 3. & 0.00 & 0.01 & & 0.00 & 0.00 & 0.00 & 0.00 & 0.01 & 0.00 & 0.89 & 0.21 & 0.99 & 0.00 & 0.01 & 0.36 \\
\hline 4. & 0.00 & 0.00 & 0.00 & & 0.07 & 0.00 & 0.00 & 0.03 & 0.01 & 0.00 & 0.00 & 0.00 & 0.00 & 0.75 & 0.00 \\
\hline 5. & 0.00 & 0.00 & 0.00 & 0.07 & & 0.00 & 0.00 & 0.08 & 0.00 & 0.00 & 0.00 & 0.02 & 0.00 & 0.72 & 0.05 \\
\hline 6. & 0.00 & 0.07 & 0.00 & 0.00 & 0.00 & & 0.94 & 0.00 & 0.00 & 0.00 & 0.02 & 0.00 & 0.60 & 0.00 & 0.00 \\
\hline 7. & 0.00 & 0.01 & 0.00 & 0.00 & 0.00 & 0.94 & & 0.00 & 0.00 & 0.00 & 0.03 & 0.00 & 0.60 & 0.00 & 0.00 \\
\hline 8. & 0.00 & 0.00 & 0.01 & 0.03 & 0.08 & 0.00 & 0.00 & & 0.00 & 0.03 & 0.00 & 0.04 & 0.00 & 0.32 & 0.43 \\
\hline 9. & 0.00 & 0.00 & 0.00 & 0.01 & 0.00 & 0.00 & 0.00 & 0.00 & & 0.00 & 0.00 & 0.00 & 0.00 & 0.07 & 0.00 \\
\hline 10. & 0.00 & 0.00 & 0.89 & 0.00 & 0.00 & 0.00 & 0.00 & 0.03 & 0.00 & & 0.22 & 0.31 & 0.01 & 0.00 & 0.53 \\
\hline 11. & 0.00 & 0.93 & 0.21 & 0.00 & 0.00 & 0.02 & 0.03 & 0.00 & 0.00 & 0.22 & & 0.13 & 0.07 & 0.00 & 0.11 \\
\hline 12. & 0.00 & 0.00 & 0.99 & 0.00 & 0.02 & 0.00 & 0.00 & 0.04 & 0.00 & 0.31 & 0.13 & & 0.00 & 0.00 & 0.59 \\
\hline 13. & 0.00 & 0.21 & 0.00 & 0.00 & 0.00 & 0.60 & 0.60 & 0.00 & 0.00 & 0.01 & 0.07 & 0.00 & & 0.00 & 0.00 \\
\hline 14. & 0.00 & 0.00 & 0.01 & 0.75 & 0.72 & 0.00 & 0.00 & 0.32 & 0.07 & 0.00 & 0.00 & 0.00 & 0.00 & & 0.00 \\
\hline 15. & 0.00 & 0.02 & 0.36 & 0.00 & 0.05 & 0.00 & 0.00 & 0.43 & 0.00 & 0.53 & 0.11 & 0.59 & 0.00 & 0.00 & \\
\hline
\end{tabular}


В этом хаосе есть определенные закономерности, но все они показывают, что доля стохастики укладывается в $k<20 \%$, и это означает крайне низкую эффективность стохастики. Эпоха традиционной науки уходит в прошлое при описании психофизиологических систем, complexity. На это обращал внимание и В. С. Стёпин, развивая представления о трех типах научной рациональности [12]. Он неоднократно говорил о непрерывном изменении вероятности $P$ (у нас - функций распределения $f(x)$ ) для биосистем, но количественного описания не создал ни Н. А. Бернштейн (он первый заговорил о повторении без повторений), ни I. R. Prigogine (он подчеркивал, что уникальные системы - не объект науки), ни W. Weaver. Только сейчас, в эффекте Еськова - Зинченко, мы можем говорить о количественных закономерностях в биомеханике: повторения треморограмм $\left(k_{2}\right)$ падают при переходе к тремору $\left(k_{1}\right)$.

Три нобелевских лауреата (M. Gell-Mann, I. R. Prigogine, J. A. Wheeler) относили complexity (эмерджентные биосистемы) к детерминированному хаосу $[19,21,23]$. Сейчас становится очевидной их ошибочность. У гомеостатических систем (СТТ) их автокорреляционные функции $A(t)$ не стремятся к нулю, нет свойства перемешивания (меры неоднородны), а константы Ляпунова меняют знак от выборки к выборке. Все непрерывно изменяется, как их $f(x)$, СПС и $A(t)$, так и их другие характеристики. СТТ - complexity в рамках современной науки невозможно описывать. На это обращал внимание и Роджер Пенроуз: "Что означает "вычислимость", когда в качестве входных и выходных данных допускаются непрерывно изменяющиеся параметры?» [11, с. 165]. В биомеханике мы не можем произвольно повторить начальное состояние $x\left(t_{0}\right)$, т. е. поставить палец в любую, конкретную точку пространства (из-за непрерывного тремора $d x / d t \neq 0$ ). Нет повторений для $f(x)$, $A(t)$ произвольно изменяются. Произвольные движения (теппинг) не являются произвольными («повторение без повторений») в плане их реализаций, т. к. они реализуются хаотически, доля стохастики не превышает $20 \%$ для $f(x)$ и $30 \%$ для $A(t)$ и $A 4 X$.

Сейчас очевидно, что все полученные факты демонстрируют особые свойства биосистем - complexity, и они являются объектом постнеклассики В. С. Стёпина [12], где непрерывно вероятность $P$, и функции распределения $f(x)$, постоянно изменяются. Статистика при разовых измерениях (выборках) не может давать объективную информацию о состоянии психофизиологических систем. Регуляция в биомеханике - это регуляция уникальных систем. У СТТ нет начального (повторяющегося) состояния $x\left(t_{0}\right)$, иx $f(x), C П C$, $A(t)$ непрерывно изменяются, и они не являются объектом современной науки. Постнеклассика В. С. Стёпина, теория хаоса-самоорганизации должны строиться на новом формальном аппарате (теории) для описания СТT. 
Доказательств получено уже достаточно (более 20000 испытуемых и более 1 млн. выборок). Любой исследователь сейчас сам может повторить опыты в биомеханике и построить матрицы парных сравнений выборок ТМГ, ТПГ, их СПС и A(t). Эффект Еськова - Зинченко является фундаментальным эффектом для всех явлений и эффектов в психологии, т. к. психика, ВНД не подчиняется законам стохастики. С нашей точки зрения, теперь использовать стохастику в разовых опытах нецелесообразно $[3,4,5,7,8,9,10]$, требуется повторение или расчет квазиаттракторов, если исследователь не может повторить опыты многократно.

\section{Квазиаттракторы как метод и модели в изучении СTT - complexity}

Сейчас разработана и построена компартментно-кластерная теория биосистем (ККТБ), которая учитывает только 1-й принцип организации СТТ (динамика поведения отдельного элемента не имеет информационного значения). Согласно ТХС, в психофизиологии сейчас имеются неопределенности 1-го и 2-го типов. Эти неопределенности полностью выводят СТТ из области современной науки и переводят биосистемы в область постнеклассики, в область глобальной неопределенности, аналогичной принципу Гейзенберга в квантовой механике, в область теории гомеостатических систем $[3,7,8,9,10]$. Эффект Еськова - Зинченко сейчас описывается параметрами квазиаттракторов (КА), которые характеризуют биомеханический гомеостаз. При этом можно идентифицировать реальные стационарные режимы, что невозможно в стохастике (там всегда $d x / d t \neq 0$ ) из-за непрерывного калейдоскопа статистических характеристик ТМГ и ТПГ (в виде $f(x)$, $A(t)$ и т. д., что представлено выше в таблицах 1, 2, 3).

Другие методы изучения, другие модели позволяют изучать неопределенности 1-го типа, когда мы имеем совпадение двух выборок, которые описывают два разных психических состояния. Иными словами, современная наука доказывает отсутствие различий, а TXС их показывает. Это уже различия в покое и движении биомеханических систем. Для моделирования этих процессов мы применили новые методы нейрокомпьютинга, когда две выборки в статистике не различаются, а нейросеть демонстрирует их различие $[13,14]$. Неопределенность 2-го типа регистрируется для всех психофизиологических параметров человека. Например, при переездах учащихся с Севера на Юг РФ и обратно сенсомоторные реакции не различаются в стохастике, а ТХC их выявляет.

Статистика показывает статику (покой), а нейрокомпьютинг и ТХС показывают различие в двух сравниваемых состояниях. Это сейчас классифицируется в TXС как неопределенность 1-го типа. При этом нейроэмулятор (НЭВМ) и ТХC решают задачу бинарной классификации - разделение двух выборок, 
которые в статистике не разделяются. Очевидно, что это требует пересмотра понятий статики (неизменности) и кинематики (изменений) в психологии и психофизиологии.

Для медицины такая ситуация катастрофична, т. к. врач не сможет отличить больной организм от здорового, не сможет выявить начало заболевания. Или наоборот, не сможет выявить начало выздоровления, т. к. с позиций статистики организм отдельного человека или обследуемой группы не отличается от здорового организма (или от исходно больного организма). Как было показано в медицине, эффект Еськова - Зинченко $[5,8]$ - это не такая уж редкая ситуация, когда применяют методы лечения, а улучшений как бы и не наблюдают. Все такие случаи могут быть разрешены с помощью нейроэмуляторов или путем сравнения параметров квазиаттракторов, которые образуют вектор состояния организма человека. Отметим, что нейроэмулятор при этом работает в особом режиме многократных итераций, что подобно работе мозга (нейросети гиппокампа). Это еще раз подчеркивает, что принципы организации живой природы можно и нужно не только изучать, но и использовать в исследовательских целях. Новые методы ТХС основаны на многократных итерациях в работе нейросети, и они реализуют принципы работы нейросетей мозга, когда мы имеем многократные повторения, но они всегда происходят без повторений (каждый раз мы получаем новую выборку весов $W_{i}$ всех диагностических признаков $x_{i}$ ). НЭВМ работает эффективно, когда мы повторяем работу нейросетей мозга!

Таким образом, методы нейрокомпьютинга устраняют неопределенность 1-го типа путем многократных итераций (число N повторов настройки нейросети в режиме бинарной классификации у нас составляет несколько тысяч, $N>1000)$. При этом возможно применение и расчетов квазиаттракторов для устранения неопределенности 1-го типа. Одновременно расчет параметров квазиаттракторов целесообразно применять и для устранения неопределенности 2-го типа. В этом случае мы имеем для испытуемого, находящегося в неизменном психическом состоянии, непрерывное изменение всех его статистических характеристик. Об этом мы говорили выше, когда выделяли непрерывное изменение функций распределения $f(x)$ для получаемых подряд выборок любых $x_{i}$, их СПС и автокорреляционных функций $A(t)$. Все статистические характеристики вектора $x(t)$ непрерывно (и хаотически) изменяются. В этом случае в ТХС говорят о неопределенности 2-го типа для получаемых подряд выборок исследуемой психофизиологической системы. Можно сказать, что неопределенность 2-го типа - это базовая характеристика всех психических и психофизиологических систем, психического гомеостаза в целом, характеристика психики человека, как СТT - complexity $[5,13,14,15,16,17,18]$. Нейросети мозга - это тоже СТТ, и любые проявления высшей нервной 
деятельности тоже будут демонстрировать эффект Еськова - Зинченко, когда выборки, их $f(x)$ неповторимы произвольно, как и их спектральные характеристики (СПС, например) или автокорреляционные функции $A(t)$.

В этом случае только параметры квазиаттракторов (объем $V_{G}$ и координаты центра квазиаттрактора, $x_{i}^{c}$, могут показать наличие неизменности психического состояния (покой) или его (квазиаттрактора) движение. В этом случае движение квазиаттрактора в фазовом пространстве состояний будет представлять эволюцию биосистемы, и эта эволюция практически не может быть зарегистрирована методами статистики (по крайней мере, ее начало). Используя методы ТХС, мы реально можем регистрировать состояние покоя биосистемы (неизменность гомеостатического регулирования) или состояние эволюции. Другими методами это выполнить невозможно. Современная наука, основанная на функциональном анализе или статистике, не может точно изучать СТТ из-за их постоянной изменчивости и отсутствия стационарных режимов в виде $d x / d t=0$ или сохранения статистических функций $f(x)$. Все изменяется при неопределенности 2-го типа, или наоборот, стохастика показывает неизменность, а реально психофизиологические параметры изменяются.

Методы ТХC (в виде итераций нейроэмулятора или расчета параметров квазиаттракторов) обеспечивают разрешение неопределенности и 1-го типа и 2-го типа. Это расширяет диапазоны диагностики нормы и патологии в психиатрии и делает психофизиологические измерения более чувствительными в оценке неизменности (или реальных изменений) параметров сложных психических и психофизиологических состояний человека. Одновременно для complexity с хаотической динамикой поведения $x_{i}$ (неопределенность 2-го типа) мы вообще не можем ничего диагностировать в рамках стохастики, и методы ТХС становятся единственными в изучении функциональных систем организма (ФСО) человека, о которых говорил П. К. Анохин [1], и в изучении эффектов «повторение без повторения» Н. А. Бернштейна [2], и в целом ряде других исследований, когда мы имеем дело с неопределенностью в системе регуляции на основе ВНД. Эффект Бернштейна с момента его открытия (1947) до настоящего времени вообще никем не изучался и никем не описывался (количественно). Сейчас мы используем для количественного описания неопределенности в психологии и психофизиологии эффект Еськова - Зинченко и методы ТХC (расчет квазиаттракторов и нейро-ЭВМ), которые устраняют неопределенности 1-го и 2-го типов [4, 5, 7, 8, 9, 10, 13, 14$]$.

Поскольку в режиме «повторение без повторений» находятся все ФСО человека, становятся понятными важность и необходимость не только изучения систем со 2-м типом неопределенности, но и их моделирование и описание. Это тем более существенно, что неопределенности 1-го и 2-го типов 
переводят психологию и психофизиологию в класс исторических наук (изучение артефактов), если мы будем использовать традиционные методы функционального анализа или статистику. Повторение без повторений, эффект Еськова - Зинченко накладывает существенные ограничения на применение стохастики в психологии.

Сейчас разработаны модели динамики поведения таких систем на основе компартментно-кластерного подхода. Оказалось, что двухкластерные, трехкомпартментные модели могут описывать динамику тремора, теппинга и даже развитие болезни Паркинсона $[5,15,16,17,18]$. В частности, они могут моделировать матрицы парных сравнений выборок, которые характеризуют тремор, теппинг, ЭМГ, ЭНГ, ЭЭГ и мн. др. процессы. Компартментнокластерная теория биосистем (ККТБ) оказалась наиболее реальной теорией в описании особого хаоса сложных биосистем, СТТ - complexity. Других методов и моделей для описания СТТ пока не создано. ККТБ является на сегодня единственной теорией, в которой можно моделировать системы третьего типа в виде матриц парных сравнений выборок, когда $f(x)$, СПС и A(t) непрерывно и хаотически изменяются $[5,15]$.

Компартментно-кластерные модели могут описывать переход ФСО от нормы к патологии и обратно (т. е. выздоровление пациента). Например, сейчас в рамках ККТБ разработаны модели болезни Паркинсона и процессы лечения этого заболевания различными препаратами (ЮMEKC, L-Допа и др.). В целом, нейроэмуляторы, расчет квазиаттракторов и компартментно-кластерный подход на сегодня являются единственными методами и моделями, которые адекватно описывают системы третьего типа; в наших примерах речь идет о биомеханике. Они описывают статику и эволюцию СТТ в ФПС, что совершенно невозможно в рамках стохастики и детерминизма.

В качестве примера представим, как можно определять реальные изменения в параметрах биомеханических систем (в частности, тремора), которые и без других внешних воздействий демонстрируют хаотический калейдоскоп изменений любых стохастических характеристик (в таблицах 1 и 3 это были $f(x)$ и их автокорреляционные функции). Если испытуемому изменить условия проведения эксперимента, например, предъявить внешний раздражитель - звук, охладить конечность перед регистрацией тремора или применить постоянно действующую силу (прикрепить груз к пальцу), то параметры квазиаттракторов сразу будут изменяться. Это будет происходить на фоне продолжающихся непрерывных изменений выборок $x_{i}(t)$, их $f(x), A(t)$, СПС и т. д.

Действительно, если к пальцу испытуемого подвесить груз $m=300$ гр., то изменяются матрица парных сравнений выборок и параметры квазиаттракторов, получаемых треморограмм. В таблице 4 мы представляем итоговые 
значения одного из более чем из 1000 опытов по регистрации параметров треморограмм в рамках ТХС. Здесь первый столбец показывает значение площади $S$ квазиаттрактора треморограммы испытуемого БАВ в спокойном состоянии (без нагрузки), а 2-й столбец показывает S и среднее значение для этой выборки $<S_{2}>$ при нагрузке конечности (пальца) массой $m=300$ гр.

Очевидно, что средняя площадь квазиаттрактора (КА) свободной конечности $<S_{1}>=0,26$ значительно меньше средней площади конечности (пальца) при прикреплении $m=300$ гр., где $\left\langle S_{2}>=1,24\right.$. Именно площади $S$ квазиаттракторов дают существенные изменения, что и показывает различия между двумя состояниями испытуемого в биомеханическом и психофизиологическом плане. Система регуляции тремора работает разным образом при удержании свободной конечности и нагруженной (массой 300 гр.), но в стохастике (путем анализа выборок) это выявить невозможно из-за неопределенности 2-го типа (см. таблицу 1).

С позиций стохастики мы при $N=15$ повторах испытаний имели бы обычный для СТТ хаотический калейдоскоп выборок, их $f(x), A(t)$, СПС и т. д. Когда все хаотически изменяется, то непонятно, какие характеристики следует использовать. Мы сейчас продемонстрировали: параметры квазиаттракторов значимо и существенно изменяются при изменении условий проведения эксперимента. Увеличение $S_{2}$ по отношению к $S_{1}$ наблюдалось у всех испытуемых при более чем 1000 проведенных испытаний. Сейчас у нас имеются огромные базы данных не только в биомеханике, но и других разделов психофизиологии и физиологии ФСО.

Таблица 4.

Значения площадей $\left(S^{*} 10^{-6}\right)$ квазиаттракторов выборок треморограмм испытуемого БАЕ с нагрузкой и без нагрузки (300 гр.), 28 лет (число повторов $N=15$, уровень значимости критерия Вилкоксона $p=0.00$, при критическом уровне значимость $p<0.05$ )

\begin{tabular}{|c|c|c|}
\hline & $\begin{array}{c}\text { Значение площади } S^{*} * 10^{-6} \text { КА без } \\
\text { нагрузки }\end{array}$ & $\begin{array}{c}\text { Значение площади } S^{*} 10^{-6} \text { KA c } \\
\text { нагрузкой (300 гр.) }\end{array}$ \\
\hline 1. & 0.23 & 1.71 \\
\hline 2. & 0.09 & 1.80 \\
\hline 3. & 0.17 & 1.86 \\
\hline 4. & 0.29 & 0.98 \\
\hline 5. & 0.26 & 0.68 \\
\hline 6. & 0.15 & 0.72 \\
\hline 7. & 0.58 & 1.03 \\
\hline 8. & 0.10 & 0.90 \\
\hline
\end{tabular}


Продолжение таблицы 4.

Значения площадей $\left(S^{*} 10^{-6}\right)$ квазиаттракторов выборок треморограмм испытуемого БАЕ с нагрузкой и без нагрузки (300 гр.), 28 лет (число повторов $\boldsymbol{N}=15$, уровень значимости критерия Вилкоксона $p=0.00$, при критическом уровне значимость $p<0.05$ )

\begin{tabular}{|c|c|c|}
\hline & $\begin{array}{c}\text { Значение площади } S^{*} 10^{-6} \text { КА безрузки } \\
\text { бае }\end{array}$ & $\begin{array}{c}\text { 3начение площади } S^{*} 10^{-6} \text { KA с } \\
\text { нагрузкой (300 гр.) }\end{array}$ \\
\hline 9. & 0.27 & 0.92 \\
\hline 10. & 0.30 & 1.82 \\
\hline 11. & 0.19 & 1.07 \\
\hline 12. & 0.18 & 0.61 \\
\hline 13. & 0.15 & 0.84 \\
\hline 14. & 0.55 & 0.95 \\
\hline 15. & 0.36 & 2.65 \\
\hline$\langle\mathrm{S}\rangle$ & 0,26 & 1,24 \\
\hline
\end{tabular}

Очевидно, что методы ТХС следует применять как при неопределенностях 1-го типа, когда все наблюдаемые статистические функции $f(x)$ совпадают до воздействия и после, так и при неопределенностях 2-го типа, когда все статистические характеристики изменяются хаотически и непрерывно. В этом случае мы имеем эффект Еськова - Зинченко в психологии, и теперь этот эффект требует изучения и изменения наших представлений о реальном хаосе систем, которые регулируются со стороны ВНД со стороны нейросетей мозга. Последние в представлении Н. А. Бернштейна (120-летие со дня рождения которого мы сейчас, в 2016 г., отмечаем) и обеспечивают «повторение без повторений». Очевидно, что добиться одинакового состояния (или динамики) в системах из миллиардов элементов (нейронов) практически невозможно. Это более фантастическая ситуация, чем демон Максвелла в термодинамике.

\section{Выводы}

1. Системы mретьего muna (СТT), о которых в 1948 г. говорил W. Weaver и которые в 1947 г. пытался описать в виде «повторений без повторений» Н. А. Бернштейн - это реальные системы в биомеханике и всей психофизиологии.

2. Для СТТ характерен эффект Еськова - Зинченко, когда все подряд получаемые выборки систем регуляции движения вместе с их статистическими функциями распределения $f(x)$, их автокорреляционными функциями $A(t)$ и др. статистическими характеристиками демонстрируют хаотический 
калейдоскоп - непрерывные изменения. Тогда любая разовая выборка с позиций стохастики произвольно неповторима (она уникальна и имеет характер исторического артефакта).

3. С позиций стохастики нет существенных различий между произвольными движениями (теппинг) и непроизвольными движениями (тремор). Однако матрицы парных сравнений выборок и параметры квазиаттракторов могут реально характеризовать произвольность (непроизвольность) движений или психическое (психофизиологическое) состояние испытуемого. Еще более выражены различия в параметрах квазиаттракторов $\left.\left(<S_{2}\right\rangle>\left\langle S_{1}\right\rangle\right)$.

4. Психология и психофизиология требуют других подходов и понятий с учетом реальности эффекта Еськова - Зинченко и представлений H. А. Бернштейна о многоуровневой системе регуляции (системы A, B, C, D) любого двигательного акта. Высшая нервная деятельность создает стохастический хаос в организации движений, который сейчас изучается в ТХС. Этот хаос можно характеризовать числом $k$ пар совпадений выборок в матрицах парного сравнения треморограмм или теппинграмм ( $k_{2}$ для теппинга всегда в 4-5 раз больше $k_{1}$ для тремора). Хаос теппинга менее выражен, чем хаос тремора $\left(k_{2}>k_{1}\right)$.

\section{Литература}

1. Анохин П. К. Кибернетика функциональных систем. - М.: Медицина, 1998. - 285 c.

2. Бернштейн Н. А. О построении движений. - М.: Медгиз, 1947. - 254 с.

3. Еськов В. М. Третья парадигма. Российская академия наук, Научнопроблемный совет по биофизике. - Самара: ООО «Офорт» (Гриф. РАН), 2011. - 250 c.

4. Еськов В. М., Газя Г. В., Майстренко Е. В., Болтаев А. В. Влияние промышленных электромагнитных полей на параметры сердечнососудистой системы работников нефтегазовой отрасли // Экология и промышленность России. - 2016. - № 1. - С. 59-63.

5. ЕСьков В. М., Еськов В. В., Вохмина Ю. В., Гавриленко Т. В. Эволюция хаотической динамики коллективных мод как способ описания поведения живых систем // Вестн. Моск. ун-та. Сер. 3. Физ. Астрон. - 2016. - № 2.

6. Еськов В. М., Майстренко В. И., Майстренко Е. В., Филатов М. А., Филатова Д. Ю. Исследование корреляции показателей функциональной асимметрии полушарий головного мозга с результатами учебной деятельности учащихся // Вестник новых медицинских технологий. 2007. - T. 14. - № 3. - С. 205-207.

7. Еськов В. М., Хадарцев А. А., Еськов В. В., Филатова О. Е. Флуктуации и эволюции биосистем - их базовые свойства и характеристики при 
описании в рамках синергетической парадигмы // Вестник новых медицинских технологий. - 2010. - Т. 17. - № 1. - С. 17-19.

8. Еськов В. М., Хадарцев А. А., Козлова В. В., Филатов М.А. и др. Системный анализ, управление и обработка информации в биологии и медицине // Том XI. Системный синтез параметров функций организма жителей Югры на базе нейрокомпьютинга и теории хаоса-самоорганизации в биофизике сложных систем. - Самара: Офорт, 2014. - 192 с.

9. Еськов В. М., Хадарцев А. А., Козлова В. В., Филатова О. Е. Использование статистических методов и методов многомерных фазовых пространств при оценке хаотической динамики параметров нервно-мышечной системы человека в условиях акустических воздействий // Вестник новых медицинских технологий. - 2014. - Т. 21. - № 2. - С. 6-10.

10. Еськов В. М., Хадариев А. А., Филатова О. Е., Хадарцева К. А., Литовченко О. Г. Проблема оценки эффективности лечения на основе кинематической характеристики вектора состояния организма // Вестник новых медицинских технологий. - 2015. - Т. 22. - № 1. - С. 143-152.

11. Пенроуз Р. «Новый ум короля: О компьютерах, мышлении и законах физики». - М.: Едиториал УРСС, 2005. - 400 с.

12. Степин B. С. Типы научной рациональности и синергетическая парадигма // Сложность. Разум. Постнеклассика. - 2013. - № 4. - С. 35-44.

13. Филатов М. А., Филатова Д. Ю., Сидоркина Д. А., Нехайчик С. М. Идентификация параметров порядка в психофизиологии // Сложность. Разум. Постнеклассика. - 2014. - № 2. - С. 4-13.

14. Филатов М. А., Филатова Д. Ю., Химикова О. И., Романова Ю. В. Метод матриц межаттракторных расстояний в идентификации психофизиологических функций человека // Сложность. Разум. Постнеклассика. 2012. - № 1. - C. 20-24.

15. Eskov V. M., Eskov V. V., Gavrilenko T. V., Vochmina J. V. Biosystem kinematics as evolution: Stationary modes and movement speed of complex systems: Complexity // Moscow University Physics Bulletin. - 2015. - V. 70 (2). pp. 140-152.

16. Eskov V. M., Eskov V. V., Gavrilenko T. V., Zimin M. I. Uncertainty in the quantum mechanics and biophysics of complex systems // Moscow University Physics Bulletin. - 2014. - V. 69 (5). - pp. 406-411.

17. Eskov V. M., Gavrilenko T. V., Vokhmina Y. V., Zimin M. I., Filatov M. A. Measurement of chaotic dynamics for two types of tapping as voluntary movements // Measurement Techniques. - 2014. - V. 57. - no. 6. - pp. 720-724.

18. Eskov V. M., Khadartsev A. A., Eskov V. V., Filatova O. E. Quantitative registration of the degree of the voluntariness and involuntariness (of the chaos) in biomedical systems // Journal of Analytical Sciences, Methods and 
Instrumentation. - 2013. - V. 3. - no. 2. - pp. 67-74.

19. Gell-Mann M. Fundamental Sources of Unpredictability // Complexity. 1997. - V. 3. - no. 1. - pp. 13-19.

20. Haken $H$. Principles of brain functioning: a synergetic approach to brain activity, behavior and cognition (Springer series in synergetics). - Berlin: Springer, 1995. - P. 349.

21. Prigogine I. The Die Is Not Cast // Futures. Bulletin of the Word Futures Studies Federation. - 2000. - V. 25. - no. 4. - pp. 17-19.

22. Weaver W. Science and Complexity // American Scientist. - 1948. - pp. 36; 536-544.

23. Wheeler J. A. Information, physics, quantum: the search for links. In Feyman and Computation: Exploring the Limits of Computers / Ed. A. J. G. Hey. Cambridge, MA: Perseus Books, 1999. - P. 309. 